\title{
Clinical significance of the prostate-specific antigen doubling time prior to and following radical prostatectomy to predict the outcome of prostate cancer
}

\author{
HISASHI TAKEUCHI ${ }^{1}$, MAKOTO OHORI ${ }^{2}$ and MASAAKI TACHIBANA ${ }^{2}$ \\ ${ }^{1}$ Department of Urology, Tokyo Medical University Ibaraki Medical Center, Ibaraki 300-0395; \\ ${ }^{2}$ Department of Urology, Tokyo Medical University, Tokyo 163-0023, Japan
}

Received September 20, 2016; Accepted December 9, 2016

DOI: $10.3892 / \mathrm{mco} .2016 .1116$

\begin{abstract}
With the advent of serum prostate-specific antigen (PSA), a larger number of prostate cancers in the early phase have been successfully detected. Although decisions to perform prostate biopsies are routinely based on PSA levels, the PSA level is easily influenced by benign prostatic hyperplasia, with poor specificity. Therefore, the aim of the present study was to assess the clinical significance of prostatespecific antigen doubling time (PSADT) prior to and following radical prostatectomy. In total, 488 patients with T1c-3N0M0 prostate cancer who underwent radical prostatectomy were included. Preoperative and postoperative PSADT were retrospectively correlated with pathological and clinical outcomes. Preoperative PSADT was measured in 204 of the 488 patients. In total, 16 out of 20 patients with a preoperative PSADT of $>24$ months had a cancer confined to the prostate compared with 105 of 184 patients with a PSADT of $<24$ months. The PSA non-recurrence rate at 5 years for patients with a preoperative PSADT of $>24$ months was significantly better compared with those with a preoperative PSADT of $<24$ months $(\mathrm{P}=0.011)$. Patients with a PSADT of $>24$ months and stable PSADT were associated with PSA recurrence following surgery, based on multivariate analysis. Postoperative PSADT was measured in 51 of 111 patients with PSA failure following surgery. Pathologically, 7 of 8 patients with a post-PSADT of $>24$ months had a cancer confined to the prostate compared with 14 of 43 patients with a post-PSADT of $<24$ months. These results suggest that patients with longer preoperative PSADTs appeared to have a favorable pathological result and a higher PSA non-recurrence rate compared with those with
\end{abstract}

Correspondence to: Professor Makoto Ohori, Department of Urology, Tokyo Medical University, 6-7-1 Nishi-shinjuku, Shunjuku-ku, Tokyo 163-0023, Japan

E-mail: mak_ohori@yahoo.co.jp

Key words: prostate cancer, radical prostatectomy, prostate-specific antigen doubling time, salvage hormonal therapy, salvage radiotherapy shorter preoperative PSADTs. A longer postoperative PSADT may facilitate the observation of patients with PSA recurrence without immediate secondary treatments.

\section{Introduction}

With the advent of serum prostate-specific antigen (PSA), a larger number of prostate cancers in the early phase have been successfully detected. Although a decision to perform prostate biopsy is routinely based on PSA levels, the PSA level is easily influenced by benign prostatic hyperplasia, with poor specificity (range, 4-10 ng/ml) (1). Furthermore, the pathological stage advances with an increase in PSA level, but considering that the range of the PSA level is so wide, the PSA level alone cannot be relied upon to determine the pathological stage. Therefore, although PSA incorporation into a nomogram provides reasonably accurate predictions of the pathological stage and PSA non-recurrence rates, novel alternative markers are required.

Previous studies on PSA kinetics, such as the PSA doubling time (PSADT), PSA slope, and PSA velocity (2-4), have indicated that these may provide valuable information for diagnosis and staging. The estimation of PSA kinetics, such as PSADT, is not difficult; however, finding an important enough reason to wait for $>3$ months to measure PSADT for staging prior to making a decision regarding the treatment strategy is difficult. Additionally, the prognostic values with respect to PSA recurrence and cancer-specific mortality remain controversial (3-7).

To clarify the clinical significance of PSADT, in the present study, the PSADTs prior to and following radical prostatectomy were investigated. Patients with PSA failure were also examined to assess how postoperative PSADT (post-PSADT) was correlated with actual salvage therapy.

\section{Patients and methods}

Patients. A total of 488 patients with clinical stage T1-3aN0M0 prostate cancer, who underwent radical prostatectomy and bilateral pelvic lymphadenectomy between January 2001 and May 2009, were retrospectively reviewed. None of the patients underwent preoperative adjuvant hormonal or radiation therapy. 
It was possible to calculate preoperative PSADT (pre-PSADT) in 204 of 488 patients by retrospectively reviewing their medical records. In these patients, PSA measurements were made more than twice prior to surgery, with $>3$-month intervals between the measurements. Clinical $\mathrm{T}$ stage was assigned based on the findings of a digital rectal examination performed using only the 2002 Union for International Cancer Control (UICC) tumor-lymph nodes-metastasis (TNM) staging system. Pathological information on biopsy and radical prostatectomy specimens was obtained from official reports.

Of the 488 patients, 111 exhibited PSA failure. PSA failure was defined as an increase in the levels of PSA by $\geq 0.2 \mathrm{ng} / \mathrm{ml}$, more than twice consecutively following surgery. Post-PSADT was calculated in 51 patients who were not immediately treated using salvage therapy.

PSADT calculation and analysis. For each patient, a linear regression of $\log$ PSA against time was calculated, and PSADT was computed as $\log 2$ divided by the slope: Ln2/rv, $\mathrm{rv}=\mathrm{d} / \mathrm{dt}\{\ln [\mathrm{PSA}(\mathrm{t})]\}$ (8). While evaluating PSADT, the value was categorized into six groups: Stable $(<0$ month) and PSA 0-6 months, 6-12 months, 12-18 months, 18-24 months, and $>24$ months.

Statistical analysis. Clinical and pathological findings and PSADT were analyzed using the Kruskal-Wallis, chi-square, and Mann-Whitney U tests. PSA non-recurrence rates were determined using the Kaplan-Meier methods, and the differences resulting from this analysis were tested using the log-rank test. Univariate and multivariate Cox regression analyses were used to predict PSA recurrence following surgery. All analyses were performed using the STATA version 10 software package (StataCorp LP, College Station, TX, USA). P $<0.05$ was considered to indicate a statistically significant value.

\section{Results}

The clinical and pathological features of the 204 patients are listed in Table I.

Pre-PSADT. The median age of 51 patients at radical prostatectomy was 68 years (range, 59-78 years). The median number of preoperative PSA measurements in 204 patients was 3.5 (range, 2-26); PSA was measured for a median period of 11.9 months (range, 3-112.8 months) prior to the diagnosis by needle biopsy. The median of the initial and final PSA levels was $5.5 \mathrm{ng} / \mathrm{ml}($ range, $0.7-46.3 \mathrm{ng} / \mathrm{ml}$ ) and $7.35 \mathrm{ng} / \mathrm{ml}$ (range, 1.1-65.5 ng/ml), respectively. The calculated median PSADT in patients with increasing PSA levels was 5.93 months (range, -28.2-287 months). PSA levels increased during the observation period in 169 of the 204 patients, whereas 35 (17.2\%) of them had stable levels.

Associations between pathological findings and pre-PSADT are shown in Table II. Prostatectomy specimen analysis revealed more locally confined cancer in patients with a PSADT of $>24$ months compared with those with shorter PSADTs $(\mathrm{P}<0.05)$.

Associations between Gleason scores and pre-PSADT are also shown in Table II. Patients with a Gleason score of 26 prostatic cancers exhibited a significantly longer PSADT
Table I. Association between clinical/pathological features and preoperative PSADT in 204 patients with clinically localized prostate cancer.

\begin{tabular}{lcc}
\hline Variable & $\begin{array}{c}\text { Frequency, } \\
\text { no. of patients }(\%)\end{array}$ & $\begin{array}{c}\text { PSADT (months), } \\
\text { median (range) }\end{array}$ \\
\hline $\begin{array}{l}\text { Last measured } \\
\text { serum PSA level }\end{array}$ & & $\mathrm{P}<0.0005$ \\
prior to surgery (ng/ml), & & \\
median (range) & & \\
$<4.0$ & $9(4.4)$ & $19.38(0-95.5)$ \\
$4.1-10.0$ & $130(63.7)$ & $7.76(-28.2-286)$ \\
$10.1-20.0$ & $43(22.1)$ & $4.02(-9.08-34.5)$ \\
$>20$ & $22(10.8)$ & $1.25(-5.0-20.6)$ \\
Clinical stage & & $\mathrm{P}=0.471$ \\
T1c & $155(76.0)$ & $6.0(-28.1-286)$ \\
T2a & $29(14.2)$ & $3.63(-9.08-44.3)$ \\
T2b & $12(5.9)$ & $8.21(0.7-82.4)$ \\
T2c & $6(2.9)$ & $1.61(0-16.9)$ \\
T3a & $2(1.0)$ & $5.1(0-10.3)$ \\
Risk group & & $\mathrm{P}<0.0005$ \\
Low & $56(27.4)$ & $9.47(-6.5-95.5)$ \\
Intermediate & $86(42.2)$ & $5.91(-28.1-286)$ \\
High & $62(30.4)$ & $3.58(-5-107)$ \\
Pathological stage & & $\mathrm{P}=0.01$ \\
pT2 & $121(59.3)$ & $6.63(-28.1-286)$ \\
pT3a & $64(31.4)$ & $5.13(-4.5-83.2)$ \\
pT3b & $16(7.8)$ & $3.23(-0.2-20.5)$ \\
pN+ & $3(1.5)$ & $1.13(0.6-8.0)$ \\
Specimen Gleason score & & $\mathrm{P}=0.098$ \\
4-6 & $55(27.0)$ & $8.86(-9.0-83.2)$ \\
7 & $94(46.0)$ & $4.59(-28.1-286)$ \\
8-10 & $55(27.0)$ & $6.0(-4.2-26.7)$ \\
\hline & &
\end{tabular}

PSADT, prostate-specific antigen doubling time.

( $>24$ months) compared with those without these characteristics ( $\mathrm{P}=0.003$; Table III).

Relationships between PSA failure following prostatectomy and pre-PSADT are shown in Table III. Pathological outcomes revealed significantly lower PSA levels in 20 patients with a PSADT of $>24$ months and stable PSADT compared with other groups. No significant differences were identified among patients with a PSADT $<24$ months (Fig. 1).

PSADTs $>24$ months and stable PSADTs were associated with PSA recurrence following surgery based on multivariate analysis with age, preoperative PSA, biopsy Gleason score, and clinical stage $(\mathrm{P}=0.008$; Table IV).

Post-PSADT. Of the 111 patients with PSA failure, 60 patients underwent salvage hormonal or radiation therapy soon after their diagnosis; therefore, post-PSADT could not be measured. The median age of 51 patients at the time of radical prostatectomy was 66 years (range, 50-77 years). The median 
Table II. Pathological features of cancer in radical prostatectomy specimens according to pre PSADT.

\begin{tabular}{|c|c|c|c|c|c|c|c|c|}
\hline \multirow[b]{2}{*}{$\begin{array}{l}\text { Pre-PSADT } \\
\text { (months) }\end{array}$} & \multicolumn{5}{|c|}{ Pathological stage, n (\%) } & \multicolumn{3}{|c|}{ Specimen Gleason score, n (\%) } \\
\hline & $\begin{array}{c}\text { No of } \\
\text { patients }\end{array}$ & pT2 & $\begin{array}{c}\text { pT3a } \\
(\mathrm{EPE}+)\end{array}$ & $\begin{array}{l}\text { pT3b } \\
(\mathrm{SV}+)\end{array}$ & $\begin{array}{c}\mathrm{pN}+ \\
(\mathrm{LV}+)\end{array}$ & $2-6$ & 7 & $8-10$ \\
\hline Stable & 35 & $17(49)$ & $14(40)$ & $4(11)$ & 0 & $8(23)$ & $19(54)$ & $8(23)$ \\
\hline$<6$ & 69 & $39(57)$ & $21(30)$ & $7(10)$ & $2(3)$ & $12(17.4)$ & $37(53.6)$ & $20(29.0)$ \\
\hline $6.0-11.9$ & 42 & $26(62.0)$ & $12(28.5)$ & $3(7.1)$ & $1(2.4)$ & $12(28.5)$ & $17(40.5)$ & $13(31.0)$ \\
\hline $12.0-17.9$ & 22 & $13(59.1)$ & $8(36.4)$ & $1(4.5)$ & 0 & $8(36.4)$ & $8(36.4)$ & $6(27.2)$ \\
\hline $18.0-23.9$ & 16 & $10(62.5)$ & $5(31.3)$ & $1(6.2)$ & 0 & $4(25.0)$ & $6(37.5)$ & $6(37.5)$ \\
\hline$\geq 24.0$ & 20 & $16(80.0)^{\mathrm{a}}$ & $4(20.0)$ & 0 & 0 & $11\left(55.0^{\mathrm{b}}\right.$ & $7(35.0)$ & $2(10)$ \\
\hline
\end{tabular}

${ }^{\mathrm{a}} \mathrm{P}=0.047 ;{ }^{\mathrm{b}} \mathrm{P}=0.003$. Pre PSADT, preoperative prostate specific antigen doubling time; $\mathrm{pT} 2$, confined prostate cancer; $\mathrm{pT} 3 \mathrm{a}$ (EPE+), extra prostatic extension positive; pT3b (SV+), seminal vesicle invasion positive; $\mathrm{LV}+$, lymph node metastasis positive.

Table III. Association between the initial PSA level and PSADT.

\begin{tabular}{lcccc}
\hline $\begin{array}{l}\text { Initial PSA } \\
(\mathrm{ng} / \mathrm{ml})\end{array}$ & $\begin{array}{c}\text { No. of } \\
\text { patients }\end{array}$ & $\begin{array}{c}\text { Preoperative } \\
\text { PSADT (month), } \\
\text { median (range) }\end{array}$ & $\begin{array}{c}\text { No. of PSA } \\
\text { measurements (times), } \\
\text { median (range) }\end{array}$ & $\begin{array}{c}\text { Period between PSA } \\
\text { measurements (month), } \\
\text { median (range) }\end{array}$ \\
\hline $0-3$ & 29 & $16.2(3-95.5)$ & $5(2-26)$ & $48.6(3.0-112.8)$ \\
$3.1-6$ & 83 & $7.4(-28.1-286)$ & $4(2-24)$ & $12.9(3.0-60.4)$ \\
$6.1-9$ & 41 & $4.8(-22.9-83.2)$ & $3(2-16)$ & $8.7(3.0-66.3)$ \\
$9.1-12$ & 24 & $3.2(-4.5-12.6)$ & $3(2-9)$ & $5.5(3.0-19.5)$ \\
$12.1-15$ & 6 & $1.9(-2.0-6.9)$ & $3.5(2-8)$ & $7.1(3.0-34.1)$ \\
$>15$ & 21 & $1.13(-5.0-13.4)$ & $3(2-6)$ & $4.6(3.1-49.0)$ \\
\hline
\end{tabular}

PSA, prostate specific antigen; PSADT, prostate specific antigen doubling time.

period from radical surgery to the diagnosis of PSA failure was 11.1 months (range, 0-125.4 months). The median preoperative serum PSA level was $8.6 \mathrm{ng} / \mathrm{ml}$ (range, 4.3-65.5 ng/ml). The median number of PSA recordings per patient following surgery was eight (range, 4-59 recordings). In total, 51 patients were clinically classified as having stage T1c $(30,58.8 \%)$, T2a $(10,19.6 \%)$, T2b $(6,11.8 \%)$, T2c (4, 7.8\%), and T3a (1, 2.0\%) tumors. The biopsy tumor grade was classified based on Gleason scores as follows: Under 2-6 (13 patients, 25.5\%), 7 (22 patients, 43.1\%), and 8-10 (16 patients, 31.4\%). In total, 21 (41.2\%) patients were identified as having locally advanced disease on final pathological examination; extracapsular extension was observed in $20(39.2 \%)$ patients, seminal vesicle involvement was observed in $8(15.7 \%)$ patients, and lymph node involvement was positive in $2(3.9 \%)$ patients. The calculated median post-PSADT was 7.76 months (range, 0.1-177.1 months). Associations among the pathological findings and post-PSADT are shown in Table IV. Pathologically, 87.5\% of the eight patients with a post-PSADT of $>24$ months, and $32.5 \%$ of the 43 patients with a post-PSADT $<24$ months had cancer confined to the prostate $(\mathrm{P}=0.004$; Table $\mathrm{V})$. No clear association was identified between the specimen Gleason scores and post-PSADT (Table V).

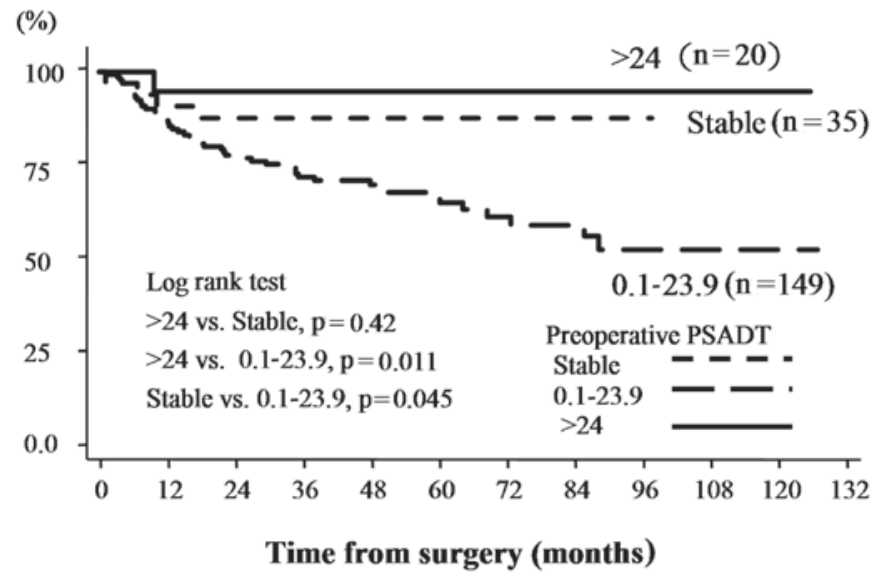

Figure 1. PSA non-recurrence rates according to preoperative PSADT. The Kaplan-Meier curve for biochemical recurrence was defined as PSA $\geq 0.2 \mathrm{ng} /$ $\mathrm{ml}$ following radical prostatectomy. PSA, prostate-specific antigen; PSADT, preoperative prostate-specific antigen doubling time.

The treatment after PSA failure is shown in Table VI. In total, 16 patients underwent salvage hormonal and/or radiation therapy. Of those 16 patients, eight underwent hormonal therapy, two underwent radiation therapy, and six underwent 
Table IV. Univariate and multivariate Cox regression analyses to predict PSA recurrence following surgery.

\begin{tabular}{|c|c|c|c|c|c|c|c|c|}
\hline \multirow[b]{2}{*}{ Variable } & & & \multicolumn{6}{|c|}{ P-value $(\mathrm{HR}, 95 \% \mathrm{CI})^{\mathrm{a}}$} \\
\hline & \multicolumn{6}{|c|}{ Univariate } & \multicolumn{2}{|c|}{ Multivariate } \\
\hline \multicolumn{3}{|l|}{ Age } & \multicolumn{3}{|c|}{ n.s. } & & \multicolumn{2}{|c|}{-} \\
\hline \multicolumn{3}{|c|}{ Preoperative PSA } & \multicolumn{3}{|c|}{$0.0029(1.03,1.00-1.060)$} & & \multicolumn{2}{|c|}{ n.s. } \\
\hline \multicolumn{3}{|c|}{ Clinical T stage } & \multicolumn{3}{|c|}{$0.001(1.68,1.23-2.29)$} & & \multicolumn{2}{|c|}{$0.009(1.60,1.12-2.29)$} \\
\hline \multirow{2}{*}{\multicolumn{3}{|c|}{$\begin{array}{l}\text { Biopsy Gleason score } \\
\text { PSA doubling time } \\
\text { (>24 months, stable, } \\
0.1-24 \text { months) }\end{array}$}} & \multicolumn{3}{|c|}{$0.002(1.44,1.14-1.81)$} & & \multicolumn{2}{|c|}{$0.005(1.38,1.10-1.75)$} \\
\hline & & & \multicolumn{3}{|c|}{$0.005(2.82,1.37-5.79)$} & & \multicolumn{2}{|c|}{$0.008(2.76,1.30-5.85)$} \\
\hline \multicolumn{9}{|c|}{ aHR, hazard ratio; CI, confidence interval. } \\
\hline \multicolumn{9}{|c|}{ Table V. Pathological stage and Gleason score according to postoperative PSADT in 51 patients with PSA recurrence. } \\
\hline \multirow[b]{2}{*}{$\begin{array}{l}\text { Post-PSADT } \\
\text { (months) }\end{array}$} & & \multicolumn{4}{|c|}{ Pathological stage, $\mathrm{n}(\%)$} & \multicolumn{3}{|c|}{ Specimen Gleason score, n (\%) } \\
\hline & $\begin{array}{c}\text { No. of } \\
\text { patients }\end{array}$ & pT2 & $\begin{array}{c}\text { pT3a } \\
(\mathrm{EPE}+)\end{array}$ & $\begin{array}{l}\text { pT3b } \\
(\mathrm{SV}+)\end{array}$ & $\begin{array}{c}\mathrm{pN}+ \\
(\mathrm{LV}+)\end{array}$ & $2-6$ & 7 & $8-10$ \\
\hline$<6$ & 23 & $2(8.7)$ & $11(47.8)$ & $8(34.8)$ & $2(8.7)$ & $0(0)$ & $11(47.8)$ & $12(52.2)$ \\
\hline $6.0-11.9$ & 13 & $8(61.5)$ & $5(38.5)$ & - & - & $3(23.2)$ & $5(38.4)$ & $5(38.4)$ \\
\hline $12.0-17.9$ & 6 & $4(66.7)$ & $2(33.3)$ & - & - & $2(33.3)$ & $2(33.3)$ & $2(33.3)$ \\
\hline $18.0-23.9$ & 1 & - & $1(100)$ & - & - & - & - & $1(100)$ \\
\hline$\geq 24.0$ & 8 & $7(87.5)^{\mathrm{a}}$ & $1(12.5)$ & 0 & 0 & $2(25)$ & $4(50)$ & $2(25)$ \\
\hline
\end{tabular}

${ }^{\mathrm{a}} \mathrm{P}=0.004$. Post-PSADT, postoperative prostate specific antigen doubling time; n, number of patients; pT2, confined prostate cancer; EPE + , extra prostatic extension positive; $\mathrm{SV}+$, seminal vesicle invasion positive; $\mathrm{LV}+$, lymph node metastasis positive.

both treatments (Table VI). None of the 15 patients with a post-PSADT $>12$ months underwent a second treatment, with a median follow-up period of 49.4 months. No association was identified between pre- and post-PSADT.

\section{Discussion}

The significance of PSADT after each treatment is an important prognostic factor (9-13). Although previous studies have indicated that the diagnostic value in patients with low PSA levels offers only limited advantages, there may be certain advantages in predicting oncological outcomes (14-17). Hanks et al (14) examined 99 patients with T1-3NXM0 prostate cancer who underwent external radiotherapy, and identified that the pre-PSADT is an independent predictor of biochemical freedom from disease. Overall, $89 \%$ patients with a PSADT of $>12$ months had no recurrence, whereas $50 \%$ of the patients with a PSADT of $<12$ months had recurrence within 1.5 years (14). Shulman et al (18) studied 163 patients with castration-resistant prostate cancer, and demonstrated that patients with a PSADT $>12$ months exhibited an improved response to deferred anti-androgen therapy compared with those with a PSADT of $<12$ months. D'Amico et al (19) examined the association between prostate cancer death and PSADT in 5,918 patients following prostatectomy, and 2,751 patients following external radiotherapy, and reported an association between a PSADT $<3$ months and death due to cancer; therefore, hormonal therapy was recommended in cases of PSA failure following surgical or radiation treatment where the patients had a PSADT of $<3$ months (19). Thus, the importance of PSADT for predicting oncological outcomes has been demonstrated, and the cutoff of PSADT may vary, depending on different populations and endpoints.

Several investigators have suggested PSADT may be used to predict treatment outcome prior to radical prostatectomy. Goluboff et al (8) examined 56 patients with three or more PSA levels prior to radical prostatectomy, and calculated the pre-PSADT; they identified that pre-PSADT did not correlate with PSA failure, final PSA, or the Gleason score, but only with the pathological stage. However, in their study, the median followup period following radical surgery was only 17.3 months, which they reported was an improvement compared with conducting a longer follow-up to examine the correlation of pre-PSADT with the pathological stage and PSA failure. Egawa et al (20) retrospectively studied pre-PSADT in 62 patients with T1-3N0M0 prostate cancer, and reported that pre-PSADT is significantly associated with the final pathological stage and PSA failure following radical prostatectomy. 
Table VI. Association between postoperative PSADT and salvage treatments.

Salvage treatments

\begin{tabular}{lccccc} 
Postoperative & No. of & & & \\
\cline { 3 - 5 } PSADT (months) & patients & Observation & Hormonal therapy & Radiotherapy & Hormonal and radiotherapy \\
\hline$<6$ & 23 & $9(39.1)$ & $6(26.1)$ & $2(8.7)$ & $6(26.1)$ \\
$6.0-11.9$ & 13 & $11(84.6)$ & $2(15.4)$ & 0 & 0 \\
$\geq 12.0$ & 15 & $15(100)$ & 0 & 0 & 0 \\
\hline
\end{tabular}

PSADT, prostate specific antigen doubling time.

Early PSA failure was revealed to be more common in patients with rapid PSADT. However, they were not able to identify any independent factor for predicting the pathological stage and PSA failure in a multivariate logistic regression analysis. The results in the present study are similar to those mentioned by Egawa et al (20). In addition, in the present paper, it has been demonstrated that pre-PSADT is an independent factor for PSA failure, similar to the results obtained for the biopsy Gleason score and clinical stage via the multivariate logistic regression analysis (Table IV).

The consensus view is that a large number of physicians consider PSADT to be useful. However, it is not popular in clinical use, mainly due to the need to wait for $>3$ months in order to calculate PSADT following cancer diagnosis, which is problematical in view of the need to select a treatment option.

In the present study, 35 patients had a stable PSADT with no change in PSA level over time. Initially, our consideration was that these patients were similar to those with a long PSADT; therefore, they should have favorable pathological results. However, the patients were revealed to have worsened pathological stages, similar to those of patients with a PSADT of $<24$ months. This is due to the fact that the majority of patients with a stable PSADT had a markedly higher initial PSA level; therefore, they underwent surgery with few PSA measurements. Consequently, the role, and importance, of PSADT where higher initial PSA levels are involved may be restricted. Conversely, in the present study, patients with a lower initial PSA level, e.g., $<4 \mathrm{ng} / \mathrm{ml}$, tended to have a longer PSADT, as shown in Table III. Also, PSADT, along with the biopsy Gleason score and clinical stage, is an independent factor for predicting postoperative PSA failure (Table IV). Therefore, the authors of the present study consider that patients with a lower initial PSA level may be safely kept under observation to measure PSADT, which may help a decision to be made on the requirement of observation without an aggressive treatment. The present study only examined cases of patients who were treated with radical prostatectomy; however, if PSADT is applied for all patients with positive biopsy, it may serve a more important role in actual clinical diagnosis. McLaren et al (6) suggested PSADT to be the strongest predictor of clinical progression in patients treated with watchful waiting. They examined 113 patients treated with watchful waiting, and reported that $\sim 40 \%$ of $\mathrm{T} 1$ patients, and $51 \%$ of $\mathrm{T} 2$ patients, had a clinical progression by 2 years, which increased to $60 \%$ at 3 years. Multivariate analysis revealed a strong correlation of PSADT with clinical progression, stage progression, and time of treatment (6).
The method for treating patients with PSA failure following radical prostatectomy remains controversial. A large number of physicians use hormonal or radiation therapy immediately after PSA failure, although certain patients may be followed up with no immediate treatments. The present study included 51 such patients. The decision to undergo no immediate treatment was suggested by their physician, who would have considered the pathological features in radical prostatectomy. However, as a result, those with a post-PSADT of $>12$ months had cancer confined to the prostate, and did not have a clinical recurrence without salvage therapy for a lengthy time period (median, 73 months). Therefore, the results of the present study indicated that, when considering early salvage therapy for PSA failure following radical prostatectomy, it is possible not to administer a salvage treatment if the cancer is pathologically confined to the prostate and PSADT is slow (e.g., >12 months).

Conversely, use of the PSADT is limited, since considering early salvage hormonal and radiation therapies is necessary if patients have prostate cancer, particularly with lymph node metastases, seminal vesicle invasion, or a Gleason score of 8-10. Our hypothesis is that it is necessary to clarify the correlation between post-PSADT and the appearance of metastasis and mortality due to prostate cancer to decide the therapy and its initiation time.

Freedland et al (21) examined 379 patients with PSA recurrence following radical prostatectomy for a median follow-up period of 10 years, and reported that clinical parameters, including PSADT, may help to identify the risk of cancerspecific mortality. However, in the present study, pre-PSADT and post-PSADT lacked any association.

The present study had certain limitations due to its retrospective nature and the small number of patients who underwent radical prostatectomy. Further investigation of the association between metastasis and mortality due to prostate cancer is currently under way.

In conclusion, a long PSADT, e.g., >24 months, indicates that the cancer is confined to the prostate, and a lower rate of PSA recurrence following surgery. This information may assist in allowing clinical decisions to be made. In particular, in prostate cancer with initial low PSA levels, the conservative therapeutic method, including active surveillance, may be considered if patients have a pre-PSADT of $>24$ months. Therefore, it is considered that measuring pre-PSADT in such patients could be informative. Furthermore, when considering early salvage therapy for PSA failure following radical prostatectomy, if the pathological stage of the specimen is cancer 
confined to the prostate with a long post-PSADT, the patient may be provided with an option of choosing observation without early salvage therapy.

\section{Acknowledgements}

The authors would like to thank Enago ${ }^{\text {TM }}$ English Editing services (www.enago.jp), for reviewing the English language of the original manuscript.

\section{References}

1. Catalona WJ, Smith DS, Ratliff TL, Dodds KM, Coplen DE, Yuan JJJ, Petros JA and Andriole GL: Measurement of prostatespecific antigen in serum as a screening test for prostate cancer. N Engl J Med 324: 1156-1161, 1991.

2. Loeb S, Roehl KA, Helfand BT, Kan D and Catalona WJ: Can prostate specific antigen velocity thresholds decrease insignificant prostate cancer detection? J Urol 183: 112-116, 2010.

3. Carter HB, Morrell CH, Pearson JD, Brant LJ, Plato CC, Metter EJ, Chan DW, Fozard JL and Walsh PC: Estimation of prostatic growth using serial prostate-specific antigen measurements in men with and without prostate disease. Cancer Res 52: 3323-3328, 1992.

4. Nam RK, Klotz LH, Jewett MAS, Danjoux C and Trachtenberg J: Prostate specific antigen velocity as a measure of the natural history of prostate cancer: Defining a 'rapid riser' subset. Br J Urol 81: 100-104, 1998.

5. D'Amico AV and Hanks GE: Linear regressive analysis using prostate-specific antigen doubling time for predicting tumor biology and clinical outcome in prostate cancer. Cancer 72: 2638-2643, 1993.

6. McLaren DB, McKenzie M, Duncan G and Pickles T: Watchful waiting or watchful progression?: Prostate specific antigen doubling times and clinical behavior in patients with early untreated prostate carcinoma. Cancer 82: 342-348, 1998.

7. Lee WR, Hanks GE, Corn BW and Schultheiss TE: Observations of pretreatment prostate-specific antigen doubling time in 107 patients referred for definitive radiotherapy. Int J Radiat Oncol Biol Phys 31: 21-24, 1995.

8. Goluboff ET, Heitjan DF, DeVries GM, Katz AE, Benson MC and Olsson CA: Pretreatment prostate specific antigen doubling times: Use in patients before radical prostatectomy. J Urol 158: 1876-1878, discussion 1878-1879, 1997.

9. Arlen PM, Bianco F, Dahut WL, D'Amico A, Figg WD, Freedland SJ, Gulley JL, Kantoff PW, Kattan MW, Lee A, et al; Prostate Specific Antigen Working Group: Prostate Specific Antigen Working Group guidelines on prostate specific antigen doubling time. J Urol 179: 2181-2185, discussion 2185-2186, 2008

10. Egawa S, Matsumoto K, Suyama K, Iwamura M, Kuwao S and Baba S: Observations of prostate specific antigen doubling time in Japanese patients with nonmetastatic prostate carcinoma. Cancer 86: 463-469, 1999.
11. Gerber GS, Gornik HL, Goldfischer ER, Chodak GW and Rukstalis DB: Evaluation of changes in prostate specific antigen in clinically localized prostate cancer managed without initial therapy. J Urol 159: 1243-1246, 1998.

12. Smith MR, Kabbinavar F, Saad F, Hussain A, Gittelman MC, Bilhartz DL, Wynne C, Murray R, Zinner NR, Schulman C, et al: Natural history of rising serum prostate-specific antigen in men with castrate nonmetastatic prostate cancer. J Clin Oncol 23: 2918-2925, 2005

13. Schmid H-P, McNeal JE and Stamey TA: Observations on the doubling time of prostate cancer. The use of serial prostate-specific antigen in patients with untreated disease as a measure of increasing cancer volume. Cancer 71: 2031-2040, 1993.

14. Hanks GE, Hanlon AL, Lee WR, Slivjak A and Schultheiss TE: Pretreatment prostate-specific antigen doubling times: Clinical utility of this predictor of prostate cancer behavior. Int J Radiat Oncol Biol Phys 34: 549-553, 1996.

15. Sandler HM, Dunn RL, McLaughlin PW, Hayman JA, Sullivan MA and Taylor JM: Overall survival after prostatespecific-antigen-detected recurrence following conformal radiation therapy. Int J Radiat Oncol Biol Phys 48: 629-633, 2000.

16. Loberg RD, Fielhauer JR, Pienta BA, Dresden S, Christmas P, Kalikin LM, Olson KB and Pienta KJ: Prostate-specific antigen doubling time and survival in patients with advanced metastatic prostate cancer. Urology 62 (Suppl 1): 128-133, 2003.

17. Semeniuk RC, Venner PM and North S: Prostate-specific antigen doubling time is associated with survival in men with hormonerefractory prostate cancer. Urology 68: 565-569, 2006.

18. Shulman MJ, Karam JA and Benaim EA: Prostate-specific antigen doubling time predicts response to deferred antiandrogen therapy in men with androgen-independent prostate cancer. Urology 63: 732-736, 2004.

19. D'Amico AV, Moul JW, Carroll PR, Sun L, Lubeck D and Chen MH: Surrogate end point for prostate cancer-specific mortality after radical prostatectomy or radiation therapy. J Natl Cancer Inst 95: 1376-1383, 2003.

20. Egawa S, Arai Y, Tobisu K, Kuwao S, Kamoto T, Kakehi Y and Baba S: Use of pretreatment prostate-specific antigen doubling time to predict outcome after radical prostatectomy. Prostate Cancer Prostatic Dis 3: 269-274, 2000.

21. Freedland SJ, Humphreys EB, Mangold LA, Eisenberger M, Dorey FJ, Walsh PC and Partin AW: Risk of prostate cancerspecific mortality following biochemical recurrence after radical prostatectomy. JAMA 294: 433-439, 2005. 